\title{
Denoising Performance of Discrete Wavelet Transform and Empirical Mode Decomposition Based Techniques on Monitoring Cardiac Electrograms From the Left-Arm
}

\author{
Gilberto Perpiñan $^{1}$, David J McEneaney ${ }^{2}$, Omar J Escalona ${ }^{3}$ \\ ${ }^{1}$ Antonio Nariño University, Cartagena, Colombia \\ ${ }^{2}$ Craigavon Area Hospital, Portadown, United Kingdom \\ ${ }^{3}$ Ulster University, Newtownabbey, United Kingdom
}

\begin{abstract}
This study evaluates the performance of three datadriven denoising approaches for enhancing the quality of cardiac electrograms recorded from bipolar leads on the left arm. An arm surface potential mapping clinical dataset was developed and used retrospectively for this study. The study arm-ECG dataset consisted of 58 cases of left-arm ECG bipolar leads gathered at the Craigavon Area Hospital, in Northern Ireland. The denoising methods considered were the Daubechies, order 10 (Db10), discrete wavelet transform (DWT), the discrete Meyer (Dmey) wavelet transform, and Empirical Mode Decomposition (EMD). Performance evaluation is quantified by measurements of the signal-to-noise ratio (SNR). The performance results on two bipolar left-arm ECG leads: Lead-1 (on the upper-arm, transversally) and Lead-4 (axially, along the upper-arm), yielded (Mean [min-max]) values for Dmey, Db10, EMD and SA of 11.8 [0.9-49.2], 13.9 [ 0,97-79.4], 17.9 [0.7-55.8] and 98.9 [1.15-649.6], respectively, for Lead-1. On Lead-4, the SNR performance results were: Dmey (6.12 [0.89-22.2]), Db10 (6.29 [0.84-19.6]), EMD (11.2 [2.19-99.2]) and SA (45.1 [4.9-200.4]). Therefore, the study revealed that the EMD based denoising technique offered the best performance.
\end{abstract}

\section{Introduction}

Dominant factors of global health are cardiovascular risk and diseases [1]. Cardiac patients account for a large proportion of attendances at hospital outpatients and emergency rooms [1]. Cardiovascular disease (CVD), is the most common underlying cause of death in the world, accounting for an estimated $31.5 \%$ of all global deaths [2]. The electrocardiogram (ECG), recorded on the body surface, has been the standard clinical tool for investigating the electrical conduction in the heart, and for its ventricular stability monitoring, by means of detecting any arrhythmic episode, and by characterising the heart rhythm in standard ECG recordings; usually with the chest leads. At risk patients present transient abnormal heart rhythms (arrhythmias) which are important in the diagnosis of heart disease and have prognostic significance [1]. As there are many different cardiac arrhythmias, their accurate detection and recording is an important clinical need and thus, continuous monitoring of the patients' heart rhythm is required for periods lasting from several days, up to a number of years, in order to enable the detection of transient arrhythmias at an early stage of heart disease, which would improve the effectiveness of appropriate treatment, reducing disease burden, disability and death. However, transient arrhythmias lasting only a few seconds are clinically difficult to detect.

There are two major categories to long-term monitoring of cardiac rhythm. a) Event recorders are noninvasive recording devices with sensing electrodes attached on the patient's body surface, usually on the chest wall; nevertheless, they are inconvenient (uncomfortable) for the patient and only suitable for short term recordings. b) Implantable loop recorders are implanted subcutaneously on the chest wall; however, while they are capable of monitoring heart rhythm during very long periods, there are considerable costs associated with the device itself and hospitalisation costs; besides the risks of surgery including infection [3]. Currently available cardiac monitoring arm/wrist bands do provide support in monitoring the heart rate; however, these cannot continuously capture ECG heart rhythm attributes provided within the heart beat P-QRS-T waveform interval. With CVD and lethal heart arrhythmias continuing to have a high mortality rate, it is important to provide patient compliant solutions for non-invasively monitoring the heart rhythm information in the P-QRS-T interval of the ECG from a long-term wearable device placed on comfortable positions of the body, such as along a single arm (armband) [4], or if possible, on the wrist. The challenges of this approach lie in the difficulty of detecting an adequate bipolar ECG signal due to the low amplitude and excessive muscle artefact in these so called "far-field ECG" locations [5].

At our Engineering Research Institute, we have 
developed various low-amplitude electrocardiographic signal recovery techniques in the past few years [4-6]. This platform of knowledge and expertise has been combined with more recent ECG denoising techniques, such as empirical mode decomposition (EMD) and enhanced EMD (EEMD), to address the aforementioned far-field arm-ECG recovery problem [6-8]. Of particular interest in this study is to comparatively evaluate the performance of three data-driven denoising techniques; based on the Discrete Wavelet Transform (DFT), EMD and having the ECG Signal Averaging (SA) method as the performance gold standard, when applied to left-arm, far-field bipolar leads electrogram signal enhancement for continuous heart rhythm monitoring.

\section{Methods}

\subsection{Bipolar Arm-ECG Leads}

In this study, two bipolar leads derived from the upper arm were used for denoising performance evaluation of the data-driven DFT and EMD techniques. Figure 1 shows the location of the BIS-QuatroTM sensor strips used in this study [9]. It describes the location of the two bipolar arm-ECG leads used. Lead-1 is transversal upperarm, bipolar lead, and Lead-4 is the axial, upper armforearm.

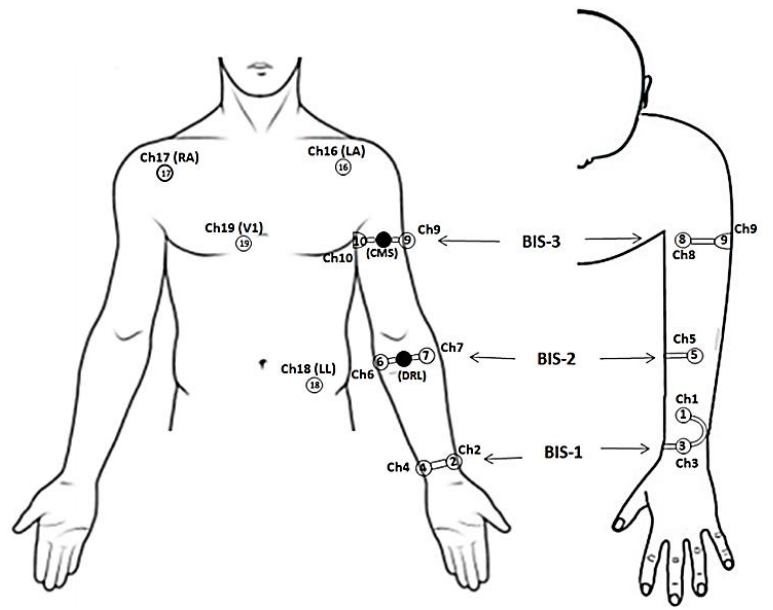

Figure 1. Unipolar left-arm potential mapping anatomical location of the 10 channels using the BISQuatroTM sensor strips; DRL is driven-right-leg; CMS is the common reference electrode [9]. Lead $1=\operatorname{ch} 9-\operatorname{ch} 10$ (Upper arm), Lead 4 = Ch6 - Ch10 (Upper ArmForearm).

\subsection{Clinical Database}

This study was carried out retrospectively from an armECG dataset of 58 subjects, aged between 19 and 82 years, whose exclusion criterion is the presence of atrial fibrillation. The database was collected in the Cardiovascular Research Unit at Craigavon Area Hospital, Portadown (Northern Ireland). Ethical approval was obtained from HSC REC B (Health and Social Care, Research Ethical Committee, reference: 16/NI/0158).

\subsection{Denoising Control Process (ECG Signal Averaging)}

The pre-filtering stage was achieved removing powerline interference of $50 \mathrm{~Hz}$ with a notch filter, then a 4 order Butterworth bandpass filter with cut-off frequencies of 1-40 Hz was implemented.

QRS complexes detection and alignment was by means of the single fiducial point (SFP) technique [8] - the zero crossing point after R-peak, in the $3-30 \mathrm{~Hz}$ bandpass filtered version of the clear standard Lead-I ECG signal (low-noise, for control) — which generated the accurate R-wave time-series, used to ensemble average a selected arm-ECG lead in a $700 \mathrm{~ms}$ signal window, centred around every time element of the SFP time-series (from $400 \mathrm{~ms}$ before the SFP and $300 \mathrm{~ms}$ after the SFP), for every armECG bipolar lead signal. Thus, in all arm-ECG bipolar leads (set of seven bipolar leads), consecutively aligned ventricular depolarization events, around 400 heart beats or more were included in the SA denoising process in each subject case [6].

\subsection{Denoising Performance}

For each recorded subject, signal-to-noise ratio (SNR) measurements from each of the selected arm bipolar leads, after the application of a data-driven signal denoising process (DWT or EMD), will be compared with SNR measurements obtained after applying the SA denoising control technique on corresponding arm bipolar leads [5-6].

SNR was computed as the ratio between the standard deviation of a signal and noise. In this case, the signal corresponds a QRS complex (120 ms) of the ECG and the noise $(40 \mathrm{~ms})$ is the segment between $\mathrm{T}$-wave end and $\mathrm{P}$ wave start (see Fig. 2).

\subsection{Denoising Using Daubechies DWT}

The noisy arm-ECG signals are subjected to 10-level DWT decomposition using the db10 wavelet bases function, where the decomposed signal can now be represented by a collection of detail coefficients (CD1CD10) and one approximation coefficient at the lowest level of decomposition (CA10) [7]. This level of decomposition was chosen because it allows for the good 
separation of both the low and high frequency content of the signal, which is important when attenuating noise artefacts with differing characteristics. In order to attenuate power line interference (PLI), the CD1-CD4 coefficients are subtracted from the signal as this has been shown in previous work to effectively suppress PLI whilst negligibly affecting the morphology of the underlying ECG signal in cases using synthesized signals [10]. The 'denoised' signal is reconstructed from the remaining detail coefficients using the inverse DWT and the level of noise attenuation achieved via this method is quantified.

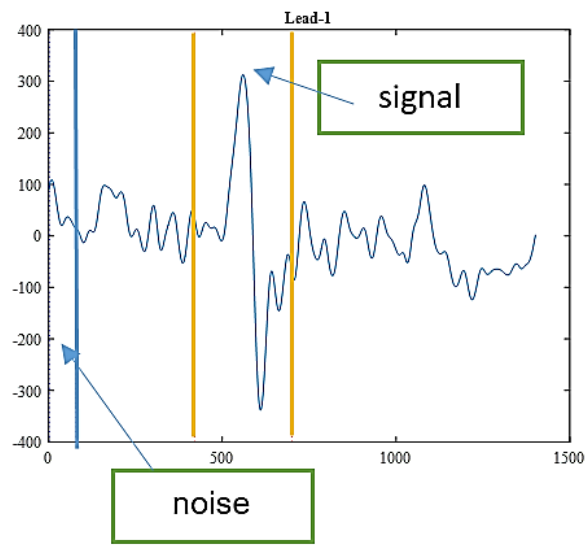

Figure 2. Positioning of the signal amplitude measuring window (120 ms) and noise amplitude measuring window (40 ms).

\subsection{Denoising Using Daubechies DWT}

The Meyer DWT (Dmey) was implemented with three levels of decomposition. CD1 and CD2 reconstructions contain entirely undesirable signal content (noise); therefore full band cancellation is required. CD3 reconstruction contains a mixture of desirable signal content and noise; therefore signal 'thresholding' can be utilized in this instance. CA1 and CA2 approximations contain low frequency information, so full band cancellation is required. ECG clean signal is the results of CD3 +CA3 [7].

\subsection{Noise Attenuation Using EMD}

Given a signal $\mathrm{x}(\mathrm{t})$, the effective algorithm of EMD can be summarized as follows:

1. Identify all extrema (maxima and minima) of $x(t)$.

2. Generate the upper and lower envelope

$\left(e_{\min }(t), e_{\max }(t)\right)$ by connecting the maxima and minima points separately with cubic spline.

3. Compute the local mean: $\mathrm{r}(\mathrm{t})=\left(\mathrm{e}_{\min }(\mathrm{t})+\mathrm{e}_{\max }(\mathrm{t})\right) / 2$.

4. Extract the detail $d(t)=x(t)-r(t)$.

5. Iterate on the residual $r(t)$.
At the end of the decomposition process, the EMD method expresses the signal $x(t)$ as the sum of a finite number of intrinsic mode functions (IMFs) and a final residual [6]:

$$
x(t)=h_{i}(t)+r_{n}(t)
$$

where $h_{i}(t)$ are the IMFs and $r_{n}(t)$ is a final residual, which is less than an arbitrarily chosen threshold.

The algorithm works iteratively by identifying the extrema of the signal and breaking it down thus ensuring that the number of modes is finite. The envelope is estimated by interpolating the extrema of the signal at each iteration. Fig.4 presents a block flowchart of the three methods denoising techniques implemented in this work.

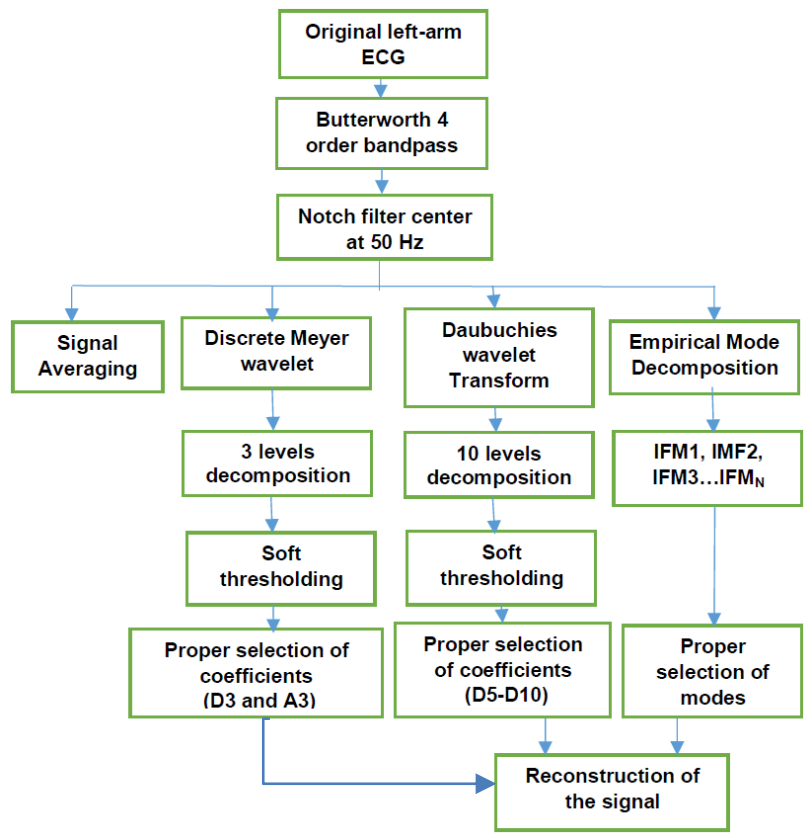

Figure 3. Block flowchart of the four main denoising processes applied to left-arm bipolar leads electrograms in this study.

\section{Results}

The results of denoising performance of the datadriven noise removal techniques and the control method (SA), are summarized in Table 1. The SNR figures are presented as the mean value and standard deviation assessed over the clinical dataset of 58 cases, for each type of denoising process and particular bipolar arm-ECG lead (Lead-1 \& Lead-4). Figures 4 and 5, graphically present the results and the performance of the denoising methods in terms of signal to noise ratio, for two left arm ECG lead. In Lead-1 SNR (media [min - max]) values for Dmey, Db10, EMD and SA are 11.8 [0.9-49.2], 13.9 [0.97-79.4], 17.9 [0.7-55.8]. 
Table 1. Results summary of SNR for transversal upperarm Lead-1, and axial upper-arm Lead-4, with signal averaging (SA), wavelets Dmey (Dmey), wavelets Daubechies $10^{\text {th }}$ order (Db10) and empirical mode decomposition (EMD).

\begin{tabular}{ccc}
\hline Methods & $\begin{array}{c}\text { Lead-1, SNR } \\
\text { (Mean [ min-max]) }\end{array}$ & $\begin{array}{c}\text { Lead-4, SNR } \\
\text { (Mean [ min-max]) }\end{array}$ \\
\hline SA & $98.9[1.15-649.6]$ & $45.1[4.9-200.4]$ \\
Dmey & $11.8[0.9-49.2]$ & $6.12[0.89-22.2]$ \\
Db10 & $13.9[0,97-79.4]$ & $6.29[0.84-19.6]$ \\
EMD & $17.9[0.7-55.8]$ & $11.2[2.19-99.2]$ \\
\hline
\end{tabular}

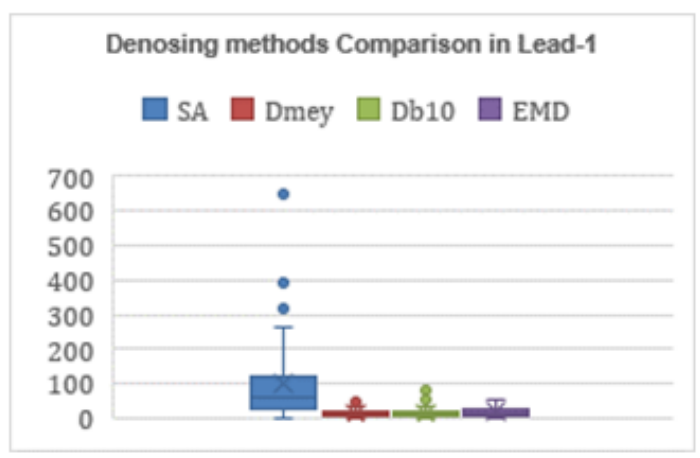

Figure 4. Denoising methods performance Boxplot for Lead-1.

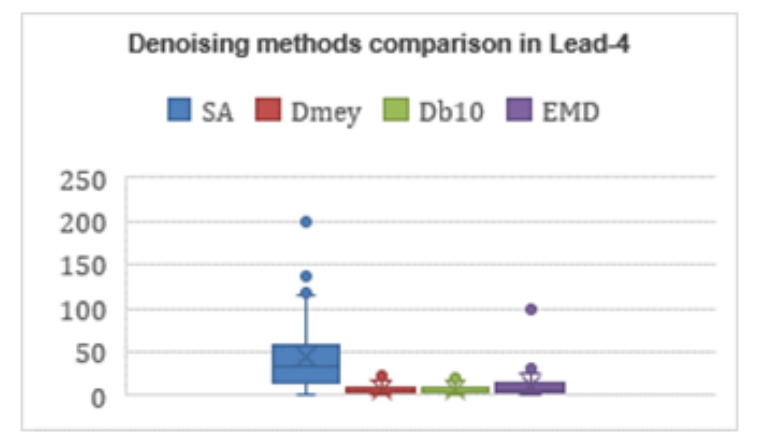

Figure 5. Denoising methods performance Boxplot for Lead-4.

\section{Discussion and Conclusion}

The electrograms from the two arm-ECG leads considered: Lead-1 (on the upper-arm, transversally) and Lead-4 (axially, along the upper-arm), were significantly enhanced as a result of the data-driven processes evaluated in this study, revealing that the EMD based denoising technique offered the best performance on both upper-arm bipolar ECG leads, in comparison to the DWT based techniques (Db10 and Dmey). The SA denoising process was far superior, but was presented as a reference in the study; it is not possible to achieve in practice. The results are in support with previous reports but with different clinical datasets, including atrial fibrillation applications (AF-ECGs) [5-7].

Acknowledgement - This research was supported by funding from the European Union (EU): Horizon 2020 Programme (WASTCArD Project, Grant \#645759).

\section{References}

[1] Benjamin, E.J., et al., "Heart Disease and Stroke Statistics 2017 Update: A Report from the American Heart Association (7/Mar/2017)", Circulation, 135:00-00, e390, 2017.

[2] Roth, G.A., Huffman, M.D., Moran, A.E., Feigin, V., Mensah, G.A. Naghavi M, et al.. "Global and Regional Patterns in Cardiovascular Mortality From 1990 to 2013. Circulation, 132:1667-1678, 2015.

[3] Lynn, W.D., Escalona, O J., McEneaney, D.J. “Arm and Wrist Surface Potential Mapping for Wearable ECG Rhythm Recording Devices: A Pilot Clinical Study". Journal of Physics: Conference Series, 450, 2013.

[4] O. Escalona, L. McFrederick, M. Borge, G. Perpiñan, J. McLaughlin, D. McEneaney, "Wrist and Arm Body Surface Cardiac Electrogram Mappping Techniques Study for Long-Term Rhythm Monitoring". In Computing in Cardiology; 44, 2017.

[5] Lynn, W.D., Escalona, O.J., Vizcaya, P.R., et al.. “ArmECG Bipolar Leads Signal Recovery Methods for Wearable Long-Term Heart Rate and Rhythm Monitoring”. In Comp. in Cardiol., 44, 2017.

[6] Escalona, O., Lynn, W., Perpiñan, G., McFrederick, et al. "Data-Driven ECG Denoising Techniques for Characterising Bipolar Lead Sets Along the Left Arm in Wearable Long-Term Heart Rhythm Monitoring", Electronics, vol. 6, no. 4, p. 84, 2017.

[7] Goodfellow, J., Escalona, O.J., Kodoth, V., Manoharan, G. "Efficacy of DWT Denoising in the Removal of Power Line Interference and the Effect on Morphological Distortion of Underlying Atrial Fibrillatory Waves in AFECG”. In IFMBE Proceedings, Jaffray D (ed.), 51, 2015.

[8] Escalona, O.J., et al., "A fast and Reliable QRS Alignment Technique for High-Frequency Analysis of the SignalAveraged ECG". Medical \& Biological Engineering \& Computing; 31: S137-S146, 1993.

[9] Vizcaya P., Perpiñan G., McEneaney D., Escalona O.J. "Standard ECG Lead I Prospective Estimation Study from Far-Field Bipolar Leads on the Left Upper Arm: A Neural Network Approach”. J. Biomed. Signal Process. \& Control, 51, 171-180.

[10] García, M., Martínez-Iniesta, M., Ródenas, J., Rieta, J.J., Alcaraz, R. "A Novel Wavelet-Based Filtering Strategy to Remove Powerline Interference From Electrocardiograms with Atrial Fibrillation". Physiological Measurement, 39(11), article number 115006, 2018.

Address for correspondence:

Name: Omar J Escalona

Full postal address: Ulster University, Shore Road,

Newtownabbey, BT37 0QB, UK.

E-mail address: oj.escalona@ulster.ac.uk 